\title{
O'ZBEK TEZAURUS LUG'ATI UCHUN SIFAT TURKUMI BAZASINI YARATISH MASALASI
}

\begin{abstract}
Abjalova Manzura Abdurashetovna
abjalova.manzura@gmail.com

Alisher Navoiy nomidagi Toshkent davlat o'zbek tili va adabiyoti universiteti dots. v.b., Filologiya fanlari bo' yicha falsafa doktori (PhD)

Elmurod Sharipov

Alisher Navoiy nomidagi Toshkent davlat

o'zbek tili va adabiyoti universiteti,
\end{abstract}

Kompyuter lingvistikasi mutaxassisligi 1-bosqich magistranti

Annotatsiya. Bugungi o'zbek tilshunosligining asosiy muammolaridan biri bu - ontologik lug'atlar yaratish masalasidir. Ushbu lug'atda o'zbek tili elektron lug'atlarining lingvistik bazasi va dasturiy ta'minotini yaratishga (sifat so $^{6} \mathrm{z}$ turkumi misolida) oid fikr va mulohazalar keltirilgan.

Kalit so'zlar: sifat, matn, asliy sifat, nisbiy sifat, so'z turkumi, tezaurus, kompyuter leksikografiyasi, sinonim, antonim, omonim.

Kirish. XXI asr - kompyuter texnologiyalari asri. Zamonaviy tilshunoslikni ham kompyuter texnologiyalarisiz tasavvur qilish qiyin. Ayni holatni kompyuter leksikografiyasida ham kuzatishimiz mumkin. Lingvistika yo'nalishida har tomonlama qulay, zamonaviy elektron lug'atlarning yaratilishi fikrimizning isbotidir. Tezaurus lug'atlar, Wordnet lug'atlar, tarjima lug'atlar, ko'p tilli elektron lug'atlarning barchasi mashina tarjimasining tayanch bazasi vazifasini bajarmoqda.

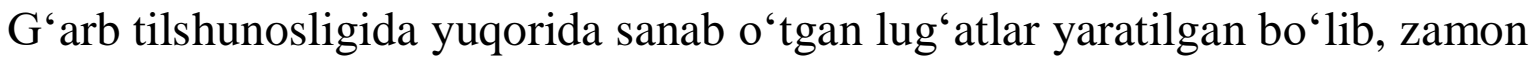
bilan hamnafaslikda rivojlantirilib borilmoqda. Biroq o'zbek tilida bu elektron lug'atlarning birontasi yaratilmagan. Hatto lingvistik bazasi ham tayyorlanmagan. Ilmiy ish darajasida biror-bir amaliy ish ham yakuniga yetkazilmagan. Xususan, tilshunosligimizda leksikografik baza yaratish masalasi o'z yechimini topmagan. Bu masala bo'yicha kichik bir tadqiqotlar, qarashlar amalga oshirilgan. Ushbu maqolada biz sifat so'z turkumi misolida ontologik lug'at yaratishni ko'rib chiqamiz.

$\mathrm{Bu}$ tipdagi lug'atlar yaratish bo 'yicha jahon tilshunosligida bir qancha ishlar amalga oshirilgan. Xususan, internet tarmog'ida bu turdagi elektron onlayn lug'atlar talaygina. Quyida ularning farqli va o'xshash tomonlarini ko'rishimiz 
mumkin:

1. https://live.babelnet.org lug'ati ko'p tilli elektron lug'atlar sirasiga kiruvchi lug'atdir.

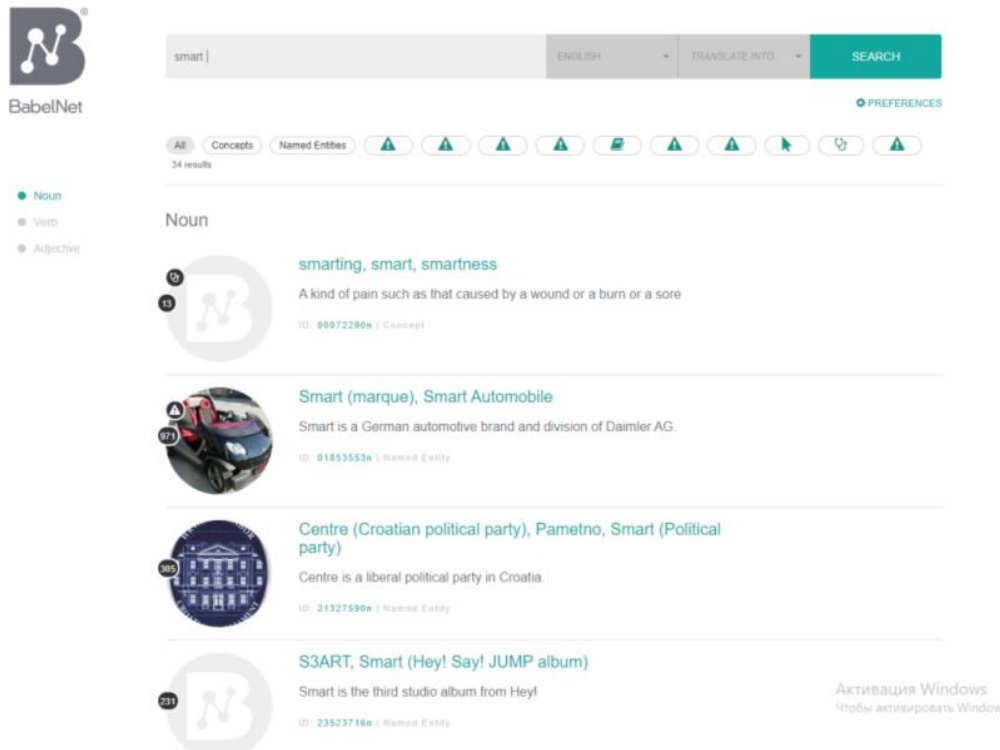

Ushbu lug'atning o'ziga xos xususiyatlari quyidagilar:

A) Lug'atdan har qanday inson onlayn foydalanishi mumkin;

B)Lug'atda 100 dan ortiq til mavjud bo'lib (o'zbek tili ham), foydalanish imkoniyati keng;

C) Qidiruv tizimining mavjudligi;

D) Qidirilayotgan so'zning izohi, sinonimi, antonimi, qaysi konseptga kirishi batafsil berilgan;

E)Har bir so'zning o"qilishi ham kiritilgan (audio);

F) So'z bildirgan tushunchaga doir rasm joylashtirilgan;

G) So'z anglatgan ma'nosiga oid namunaviy matn ham keltirilgan;

H) Asosiysi, qidirilayotgan so'zning boshqa tildagi tarjimasini onlayn ko'rish imkoni mavjud;

2. https://www.dictionary.com ham onlayn ontologik lug'atlar sirasiga kiruvchi ommabop lug'atlardan biri hisoblanadi. 


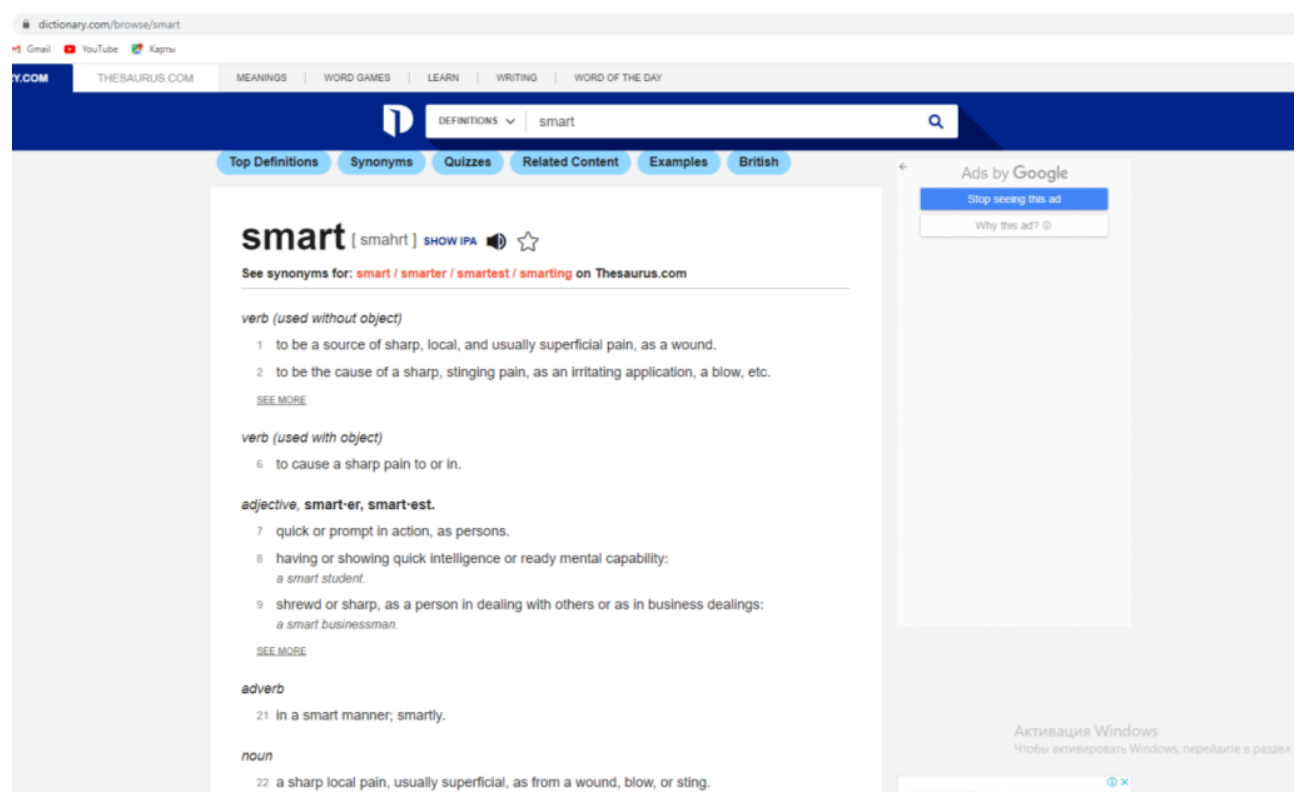

Ushbu lug'atning o'ziga xos xususiyati quyidagilar:

A) Onlayn foydalanish imkoniyati

B)Qidiruv tizimining mavjudligi;

C)Qidirilayotgan so'zning izohi, sinonimi mavjudligi

D) Har bir so'zning o'qilishi ham kiritilgan (audio shaklida);

E)Qaysi so'zlar bilan birikma hosil qilish yoki hosil qila olmasligi ko'rsatilgan

3. https://www.thesaurus.com sayti ham onlayn foydalanish

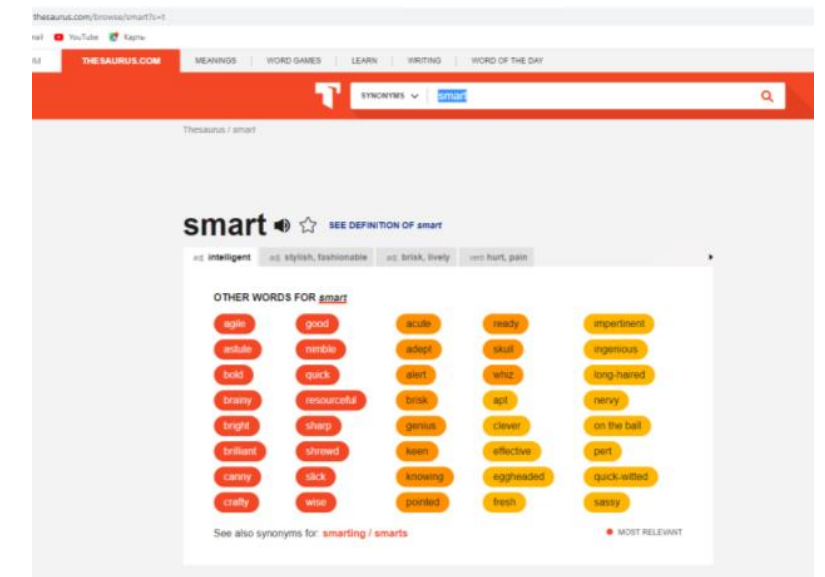

A) Onlayn foydalanish imkoniyati

B)Qidiruv tizimining mavjudligi;

C)So'zlarni ovozli shakli kiritilganligi

D) Sinonim so'zlar keltirilganligi;

E)Qo'llanilishi bo'yicha saralanganligi.

https://wordnet.princeton.edu/ Princton universiteti tomonidan ishlab chiqilgan ingliz tilining lingvistik qidiruv tizimi bo'lib, ma'lumotlar bazasi va 
interfeysi foydalanishga qulay qilib ishlangan. Bu tizimning o'ziga xos jihatlari:

A) Onlayn qidiruv mavjudligi;

B) So'zning ko'p ma'noliligiga ko'ra topib berishi;

C)Har bir ma'nosiga berilgan link orqali ko'proq ma'lumot olish mumkinligi;

D) Har bir so'zga namunaviy gap berilganligi.

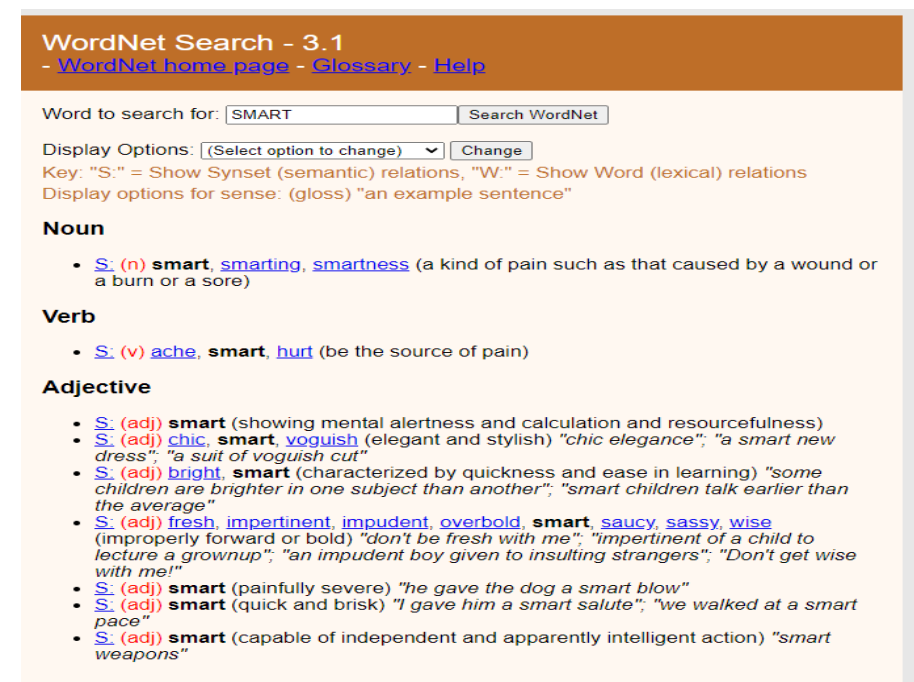

O`zbek tili ontologik bazasini yaratishda yuqorida tanishib chiqqan bir nechta bazalarni umumlashtirib, ularning har biridan foydalangan xolda ish olib kerak. Sifat so'z turkumi misolida ko'radigan bo 'lsak, quyidagi jadval holida baza tuzib olishimiz kerak.

\section{Masalan:}

«Chiroyli» sifatining ontologik lug'atini yaratib ko'ramiz:

Turkumi: Sifat;

Sinonimik qatori: go'zal, latofatli, xushro'y, xushbichim, ko'rkam;

Antonimi: xunuk, badbashara;

Omonimi: yo'q

Paronimi: yo'q;

Yasalishiga ko 'ra: sodda yasama sifat;

Turiga ko 'ra: Asliy sifat;

Bog 'lanadigan so 'zlari: Ot so'z turkumidagi barcha so'zlar;

Qaysi tildan kirib kelgan: turkiy so'z. Misollar: Shaharda shunday chiroyli qizlar bor - ko'rsang og'zing ochiladi. «Yoshlik»

Shu shaklda o'zbek tilida mavjuda bo'Igan sifatlarning har biriga to xtalib o'tamiz. Ilmiy ishda asosiy manba sifatida «Izohli lug'at»dan foydalansak, qo'shimcha manbalar sifatida badiiy va ilmiy asarlardan foylaniladi. 
Yuqorida aytib o'tganimizdek, ushbu lug'atni tayyorlash ikki bosqichdan iborat. Avvalo, lug'atning lingvistik bazasi yig'iladi. Lingvistik bazaning tarkibi esa quyidagicha:

1. So'zlar so'z turkumlarga ajratilgan bo'lishi;

2. Turkumlarning o'zi ham grammatik kategoriyalarga tasniflanishi;

3. So'zlarning izohi kiritilishi;

4. Leksik munosabatga kirisha oladigan so'zlarning birikmali va gap holatidagi bazasi yaratilishi;

5. Har bir so'z konseptual-semantik jihatdan guruhlarga biriktirilishi;

6. Sinonim, antonim, omonim so'zlarning elektron bazasi bo'lishi kerak.

Sanab o'tilgan baza yig'ilgandan so'ng ular ma'lumotlar bazasida Id kodlar bilan o'zaro bog'liq holatda biriktiriladi va dasturi yaratiladi.

\title{
Foydalanilgan adabiyotlar:
}

\author{
1. https://WordNet.princeton.edu \\ 2. https://live.babelnet.org \\ 3. http://www.roget.org \\ 4. $\mathrm{O}^{6}$ zbek tilining izohli lug'ati \\ 5. https://scienceforum.ru/2015/article/2015009933
}

